\title{
Prevention of illegal transportation using ANPR and biometric fingerprint in mining industry
}

\author{
Debi P. Tripathy*, Yelipeta N. Harish \\ Department of Mining Engineering, NIT, Rourkela 769008, India
}

Corresponding Author Email: debi_tripathy@yahoo.co.in

https://doi.org/10.18280/mmep.050318

Received: 12 June 2018

Accepted: 4 September 2018

\section{Keywords:}

automatic number plate recognition, mining, finger print, , microcontroller, Gabor filter

\begin{abstract}
Security in mining is a primary concern, which adversely affects the production cost. An efficiently detected and deterred theft can maximize the profitability of any mining organization. Many illegal transportation cases were registered in spite of provisions imposed by central and state governments under Section 23 (c) of MMDR Act 1957. This paper proposes the use of an automated checkpoint gate based on license plate recognition and biometric fingerprint system for vehicle and driver authentication vehicle tracking. Vehicle license number plate is captured using a digital camera, and the characters of the number plate are segmented using Gabor filter. A track-sector matrix is generated by considering the number of pixels in each region and was matched with existing template to identify the character. The fingerprint scans the finger and matches with the template created at the time of fingerprint registration at the machine. The checkpoint gate is closed or opened based on the output provided by the microcontroller.
\end{abstract}

\section{INTRODUCTION}

The mining industry is one of the major contributors to the growth of Indian economy. It has an average GDP of INR 686.08 billion from 2011 to 2015 [1]. The mining industry, however, faces a significant number of challenges regarding productions cost and crime. The theft of precious minerals from the mines results in huge loss to the company. Madhya Pradesh State registered 982 illegal transportation cases during the year 2013 and recovered fine worth Rs 7.11 crores [2]. Measures are being taken by the central and state governments by framing rules under section 23 (c) of MMDR Act, 1957. It was said that every truck/dumper carrying minerals should have a transit pass issued by the authorities and action would be taken against vehicle without a valid and countersigned transit pass [3].

Mahanadi Coalfields Ltd. implemented a GPS-based tracking system for the trucks, and in 2015, 36 third-party contractor's trucks were blacklisted, each carrying up to 17 tons of coal. The company installed CCTV cameras at shipment sites, hooked up trucks with satellites and fitted them with radio frequency identification tags to automatically transfer data to a control room at the company's headquarters [4]. In Northern Coalfields Limited (NCL), GPS-based vehicle tracking system with RFID identification for vehicles having integration with weighbridges was introduced to automate the coal dispatch to the end user without human interface. Through the system, an alert will be generated if any violation occurs from the specified path like a deviation from the specified path, undue stoppage, etc. and the alert will be sent to the respective in-charge of coal transportation/dispatch for taking action. GPS/GPRS device fitted in the vehicle transmits information (such as vehicle ID, latitude, longitude, date time, etc.) through GPRS network to a designated central server. The data collected in the server are plotted on the GIS map [5]. South Eastern Coalfields Limited (SECL), Bilaspur proposed an RFID-based system to identify trucks at each entry/exit gate, automates the operation of a boom barrier at the entrance gate that allows entry, and logs individual truck movement and trips into and out of the mining area [6].

Image and video processing play a significant role in the development of technologies for dealing with security issues: surveillance cameras for crime reduction and image analysis tools in the forensics field. Automatic Number Plate Recognition (ANPR) system is an important technique, used in Intelligent Transportation System. ANPR is an advanced machine vision technology used to identify vehicles by their number plates without direct human intervention. Biometrics is usually associated with the use of unique physiological characteristics to identify an individual. The application which most people associate with biometrics is security.

The present study provides an innovative approach to reducing the illegal mineral transportation based on combined ANPR and biometric fingerprint system. A hardware setup for open and close of the control gate was made by capturing the fingerprint of the driver and number plate. Microcontroller was used for initiating the control gate to open or close based on the authenticity of the drive and the vehicle.

\section{LITERATURE REVIEW}

In the literature, various license plate detection algorithms were implemented and proposed by different investigators and are summarized below:

Ozbay and Ercelebi (2005) proposed extracting the plate region and segmentation of characters from the number plate using smearing algorithms. The results proved to be $97.6 \%$ accurate for the extraction of plate region, $96 \%$ accurate for the segmentation of the characters and $98.8 \%$ accurate for the recognition and the overall system performance was $92.57 \%$ accurate [7]. 
Ravi et al. (2009) proposed fingerprint recognition using Minutia Score Matching strategy (FRMSM). To preserves the quality of the image and extract the minutiae from the thinned image block filters were used. The proposed method FRMSM gave better false matching ratio (FMR) values compared to the existing method [8].

Kulkarni et al. (2009) worked on the identification of number plate for Indian conditions. The framework consists of the integration of algorithms like: 'feature-based number plate localization' for locating the number plate, 'image scissoring' for character segmentation and statistical feature extraction for character recognition, are mainly designed for Indian number plates. The framework can recognize single and double line number plates under varying illumination conditions with a success rate of $82 \%$ [9].

Gnanasivam and Muttan (2010) proposed a productive preprocessing calculation to accomplish great vertical orientation and high ridge curvature area and flow zone around the center point for the fingerprint validation and examination. The proposed algorithm was tested on a line based highlight extraction calculation with a substantial internal database and samples of unique Finger Impression Verification Competition (FVC). The proposed algorithm resulted in 94\% accuracy for the low-quality pictures and broken edges [10].

Zhai et. al. (2010) proposed number plate recognition by morphological open and close operations and various structural elements like rectangular shape and diamond shape were operated to take out the non-plate region. They have achieved $98.7 \%$ of detection rate which was more than other methods [11].

Bana and Kaur (2011) had studied and implemented a unique finger impression acknowledgment framework based on Minutiae based matching. The approach mainly involves extraction of minutiae points from the sample fingerprint images and then performing fingerprint matching based on the number of minutiae pairings among two fingerprints. The results indicated that the proposed algorithm was not very robust and was vulnerable to effects like scaling and elastic deformations [12].

Kolour and Asadollah (2011) studied and evaluated six LPD algorithms techniques dynamic programming, Hough transform, Gabor transform, Morphology-based, AdaBoost, and Edge-based models. The results showed that the dynamic programming algorithm was the fastest and the Gabor transform was the most accuracy algorithm [13].

Asthana et. al. (2011) proposed a method for the vehicle number plate recognition using the back propagation algorithm. A number of hidden layers are proportional to the number of epochs. The authors had obtained $96.53 \%$ average recognition rate using double hidden layer and 94\% using single hidden layer [14].

Sharma and Kaur (2011) proposed Automatic Number Plate Extraction and Character Segmentation for Indian vehicles. The proposed algorithm consists of three major parts: preprocessing of image, extraction of plate region and segmentation of characters. For preprocessing of the image, histogram equalization was applied. For extracting the plate region, edge detection algorithm and various morphological operations was used. Character segmentation was done by using connected component and bounding box method. The accuracy of the algorithm for license plate extraction was $91.02 \%$ and for segmentation was $88.46 \%$ accurate [15].

Gill and Kaur (2012) presented number plate detection based on edge detection by using Sobel operator, along with dilution and template matching methods and has achieved $95 \%$ accuracy [16].

Rani et. al. (2012) proposed pixel distribution density for the image character and segmentation from the vehicle license plate. This method has a better efficiency of the total system and eliminates the problem of similarity elements like T, I, 1, $\mathrm{J}$, etc. by exploiting the small but important differences among them [17].

Aggarwal and Aggarwal (2013) performed number plate recognition by using row and column profile of the image and license plate extracted by the positioning of row and columns. The result was obtained for $1200 * 1600$ sized color images under various environmental conditions like cloudy, sunny and daytime [18].

Patel et al. (2013) discussed different ANPR techniques by considering image size, the success rate and processing time as parameters. The comparison results showed $95.6 \%, 96.5 \%$ and $98.7 \%$ success rate with ANN and self-organizing (SO) recognition, PNN, and Open source OCR Tesseract respectively [19].

Karthikeyan et al. (2013) proposed novel moves toward segmentation of characters of license plate using Connected Component Analysis (CCA). Binary thresholding method was used to separate object and background. Morphological methods were used to achieve the detection of the license plate. CCA scans and labels the pixels of a binarized image into components based on pixel connectivity Quantization and glossy compression was obtained by compressing a range of values to a single quantum value [20]

Sonavane et al. (2013) proposed a technique for an invariant system that works under different lighting conditions while capturing the images of the vehicles. Enhanced shadow removal algorithm and Connected Component Analysis method for number plate localization \& segmentation. The authors had used Skeletonization method for feature extraction and Support Vector Machine for character recognition [21]

Mohammad et al. (2014) represented an algorithm for implementation of Optical Character Recognition (OCR) to convert typewritten/handwritten images into electronically editable format by preserving font properties. The author had proposed pattern matching for optical character recognition and the experiment results showed the recognition rate for character images of the same font used of upscaling is almost $100 \%$ accurate and $70-75 \%$ accurate for noisy images [22].

Kaur and Ameeta (2014) presented the variation of Fast Fourier Transform on fingerprint recognition by fast fingerprint minutiae extraction and recognition. This approach improved the clarity of the ridge and valley structures of the input fingerprint images based on the frequency and the orientation of the local ridges and thereby extracting correct minutiae. The author combined many methods to build a minutia extractor and a minutia matcher. The results indicated variation in FFT values produced 100\% accuracy [23].

Kaur and Garg (2014) proposed the technique of recognition of number or characters from vehicle number by feature extraction and recognition with two classifiers -Back propagation neural network and k-means on text and numeral objects of number plates. The experiment resulted in $95.6 \%$ and $83.8 \%$ accuracy for the neural network and k-means classifier respectively[24].

Chouhan and Govindan (2014) had demonstrated a characteristic based method for Indian number plates that can segment number plate even if it was in any corner of the image. Their method can process number plate of any color and even 
with text vulnerability and stickers. The localization of number plate is done by using inverted ' $\mathrm{L}$ ' masking and reverse ' $\mathrm{L}$ ' filter and white pixel density. Through this process, they have achieved $90 \%$ accuracy [25].

Sharma (2015) carried out a systematic study of existing ANPR according to features used. An algorithm for localization of yellow colored license plates using morphological operations was proposed. Character segmentation was carried using histogram and intensity projections. Optical character recognition was performed using template matching. Number plate validation using excel database to control a real-time gate/buzzer with LCD and notification on email for invalid vehicles was proposed [26].

Kumar et al. (2015) proposed a novel touchless fingerprint sensing device capturing three distinct strategies at one time. The proposed an innovative touchless fingerprint sensing device capturing three different techniques at one time. For this experiment, three images, frontal, left and right finger were captured. To reduce the effect of perspective distortion, the region with minimal ridge interval variations was selected in a final mosaic image. The results indicated real minutiae and vast quality area for collected images. The side view images gave more matched minutiae than the frontal view images. The proposed method reduced the view difference problem and increased the usable area of a touchless fingerprint image [27].

Rajput (2015) correlated matching algorithm for number plate recognition for an image with $512 * 512$ size. The edge detection was done by image dilation and image erosion. The correlated coefficient matrices were used for matching the number plate with template library [28].

\section{METHODOLOGY}

The main steps involved in the proposed method are: Automatic Number Plate Recognition (ANPR), Biometric Fingerprint System and Gate Control. The block diagram of the system is shown in Figure 1.

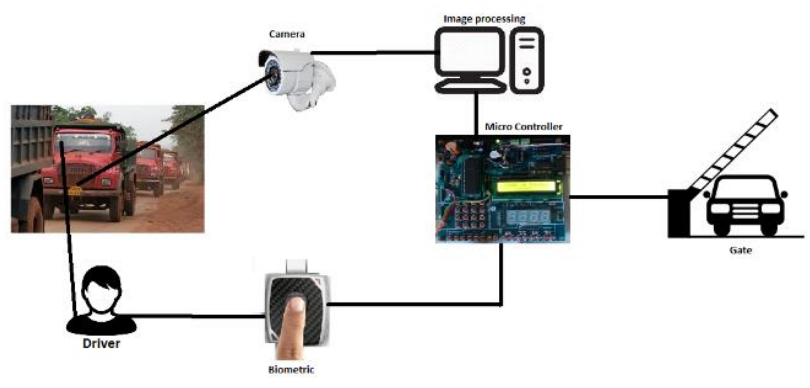

Figure 1. Block diagram of the system

\subsection{Automatic number plate recognition (ANPR)}

Image-processing technology based automatic number plate recognition can be used to identify vehicles by their license number plates. ANPR is divided into 4 steps: Image Acquisition, License number plate extraction, Character segmentation and Character recognition as shown in Figure 2.

\subsubsection{Image acquisition}

Digital camera was used for capturing the images of the vehicles. Camera was placed on a road to capture the number plates of the vehicle. The captured images were processed using Matlab software.

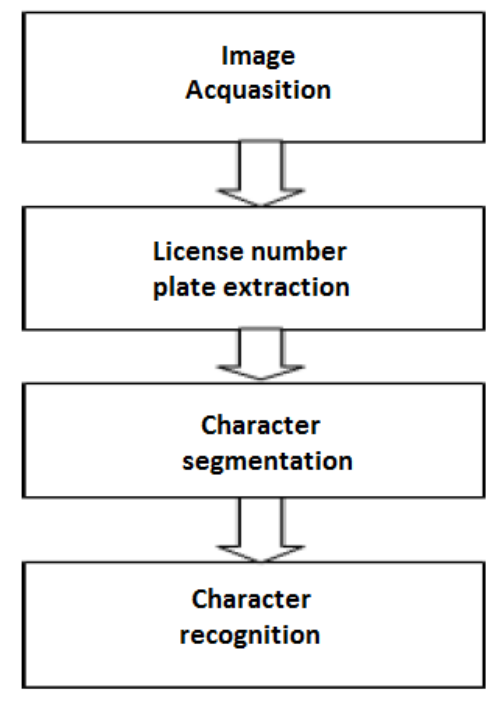

Figure 2. ANPR system

\subsubsection{License number plate extraction}

The captured images were converted into gray scale images. The noise in the images was removed by using low pass filter. The unwanted edges in the image were removed by using adaptive threshold method. The required region is segmented using threshold [29].

\subsubsection{Character segmentation}

Character segmentation was performed to partition the number plate into its individual characters. The factors that were considered during character segmentation were the noise of the image, plate frame, rivet, rotation of the plate and variance in illumination. Gabor filter was used for segmentation of number plate. This analyses the texture in every direction and scale [30].

\subsubsection{Character recognition}

The segmented individual character was scanned initial for the darker pixel at the top corner of the first character and also for the darker pixel at the bottom corner with leaving space between each character. The characters were determined by scanning from top to bottom and from left to right.

The extracted features were compared with templates by dividing the extracted character into sectors and tracks. Accordingly, a track-sector matrix was generated by considering a number of pixels in each region as shown in Figure 3. This track-sector matrix was matched with existing template to identify the character as shown in Figure 4. The existing template consists of each track-sector intersection value, track value and sector value. If all these parameters are found to match with the template values then the resultant is the character identified.

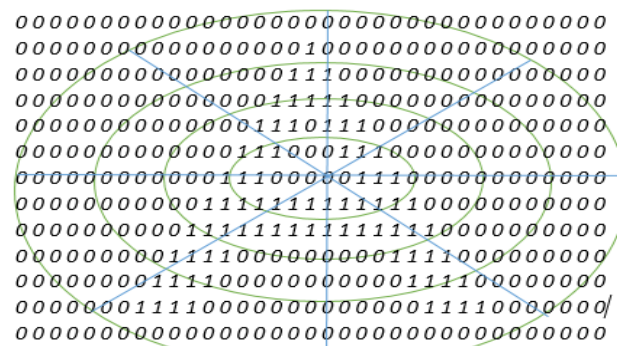

Figure 3. A track-sector matrix 


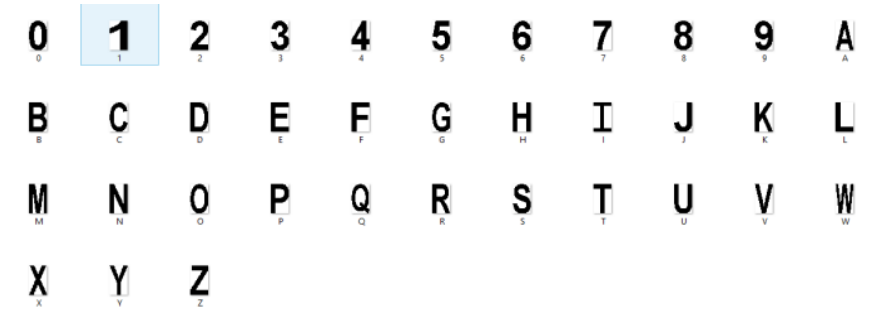

Figure 4. Existing templates

\subsection{Biometric finger print system}

An image processing based Biometric fingerprint identification can be used to identify a person. The fingerprint identification process was classified as fingerprint capturing and preprocessing, image thinning, minutia extraction, and minutia template matching [31].

\subsubsection{Fingerprint capturing and preprocessing}

A fingerprint sensor was used to capture a digital image of the fingerprint pattern. The captured fingerprint images were converted into a grayscale image that has various pixel values ranging from 0 to 255. A gray level fingerprint image contains narrow ridges separated by narrow valleys. The local ridge characteristics and their relationships were used to determine the unique nature of fingerprint.

\subsubsection{Image thinning}

The binarized image has thicker ridgelines that take more data for operation and increases the complexity while distinguishing valleys and ridges. Hence the image is thinned by using image dilation and image erosion.

\subsubsection{Minutia extraction}

A ridge is classified as the ridge that ends is termination or ridge end and the bifurcated ridge is called bifurcation. Minutiae consist of these two basic types, ridge end and bifurcation. These two categories of ridges are considered as the minutiae points as shown in Figure 5. Minutia is extracted by utilizing templates of $3 * 3$-pixel window. If the central pixel has value 1 and has only three one-valued neighboring pixels, then the central pixel is considered as ridge branch/ridge pixel. If the central pixel has value 1 and has only one one-valued neighboring pixel, then the central pixel is considered as ridge ending/termination point. If both the top-most pixel has value 1 and the right-most pixel has value 1 and they have another neighboring pixel outside the $3 * 3$ pixel window and those two pixels are considered as branches/ bifurcation point as shown in Figure 6 [8].

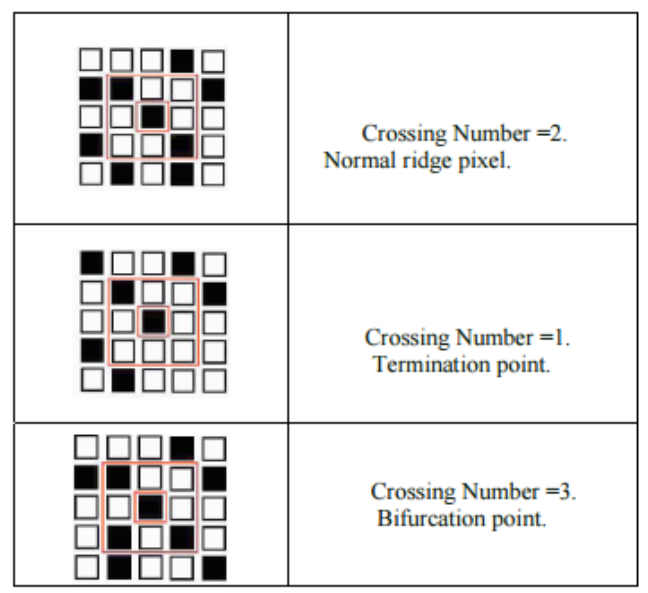

Figure 6. Crossing number and type of Minutiae

\subsubsection{Minutiae matching}

Minutiae matching involves matching the template image with the input image as shown in Figure 7. Template image was collected during enrolment and saved in the database. During recognition phase, the input image was compared against template image and decides whether the two images are from the same finger or not.
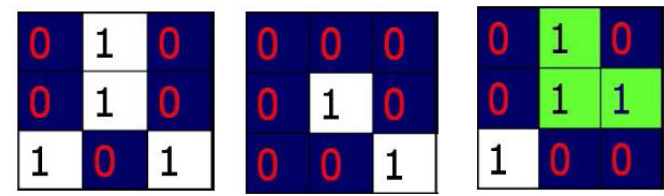

Figure 7. Templates of fingerprint [12]

\subsection{Validation and Acknowledgement}

The biometric fingerprint recognizer module was directly connected to a microcontroller using a TTL UART interface. The fingerprint captured was matched with the fingerprint stored in the database. If any of the fingerprint was matched, then the micro-controller takes response of the biometric as binary 1 and if not matched then binary 0 .

The number plate was matched with the character templates to recognize the character of the number plate. If the whole number plate matches with the number stored in the database, then the binary 1 is sent to the microcontroller and binary 0 if templates are not matched.

These binary outputs from the both the processes are taken by the micro-controller and logical AND operation was done. The output from the logical AND operation was given to the motor driver to initiate the gate as shown in Table 1 .

Table 1. Output from the microcontroller based on the ANPR and biometric module

\begin{tabular}{cccc}
\hline $\begin{array}{c}\text { O/P } \\
\text { from } \\
\text { ANPR }\end{array}$ & $\begin{array}{c}\text { O/P from } \\
\text { biometric }\end{array}$ & $\begin{array}{c}\text { Logical AND } \\
\text { operation by } \\
\text { microcontroller }\end{array}$ & $\begin{array}{c}\text { Output at } \\
\text { the gate }\end{array}$ \\
\hline 0 & 0 & 0 & $\begin{array}{c}\text { GATE IS } \\
\text { CLOSED }\end{array}$ \\
0 & 1 & 0 & $\begin{array}{c}\text { GATE IS } \\
\text { CLOSED } \\
1\end{array}$ \\
1 & 0 & 0 & $\begin{array}{l}\text { GATE IS } \\
\text { CLOSED } \\
\text { GATE IS } \\
\text { OPENED }\end{array}$ \\
\hline
\end{tabular}




\section{RESULTS AND DISCUSSIONS}

For this study, a hardware model as shown in Figure 8 with ATMEGA16 micro controllers, DC motor for gate control, a webcam for ANPR and biometric fingerprint R305 module were used. The images of the vehicles were captured using a digital camera. The image of the vehicle is shown in Figure 9.

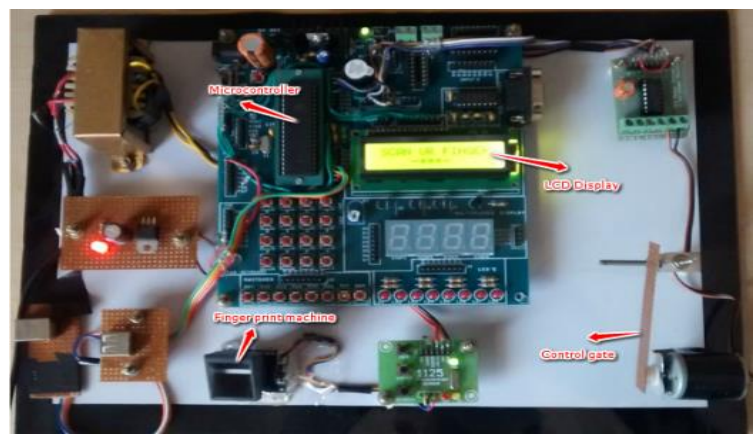

Figure 8. Hardware module

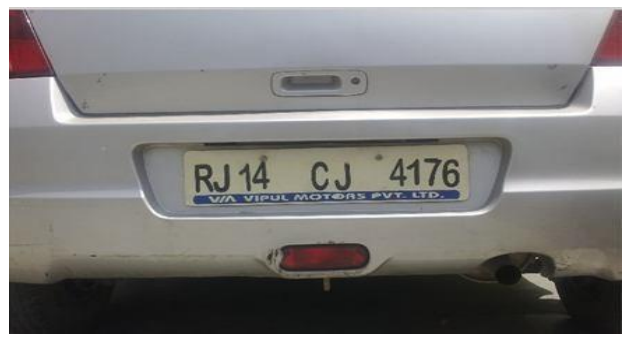

Figure 9. Image of vehicle number plate

The image captured was converted into a greyscale image as shown in Figure 10. Adaptive threshold method was used to remove the unwanted edges as shown in Figure 11. Every character was segmented from the image as shown in Figure 12. The features extracted from the segmented character were matched with the template shown in Figure 4.

Unwanted edges. The R305 fingerprint module was used for fingerprint recognition. The fingerprint scans the finger and matches with the template created at the time of fingerprint registration at the machine.

The ANPR and biometric fingerprint match the images and the binary output from the both the processes were sent to the microcontroller and logical AND operation was done. The output from the logical AND operation was given to the motor driver to initiate the gate and the acknowledge display on LCD as shown in Figure 12.

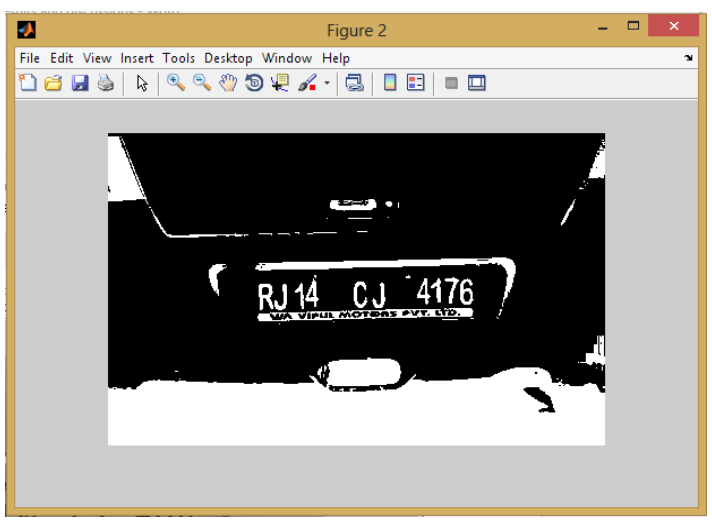

Figure 10. Greyscale image

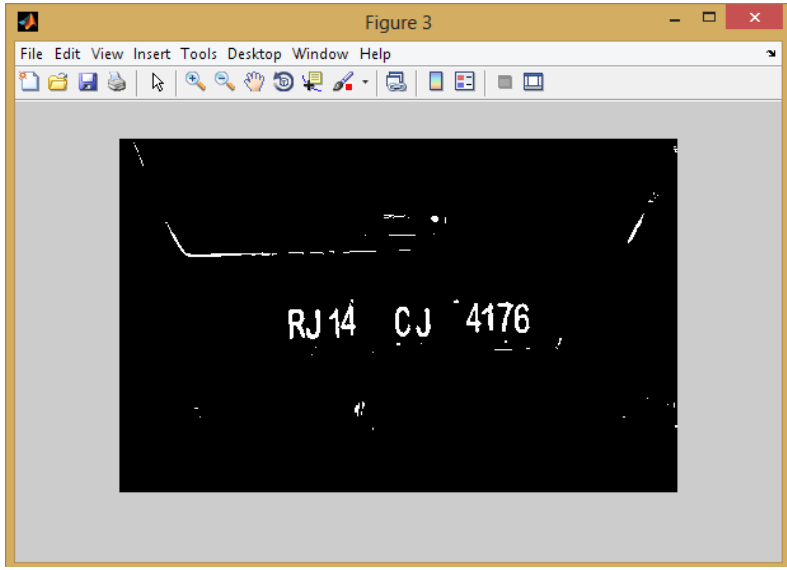

Figure 11. Image after removing

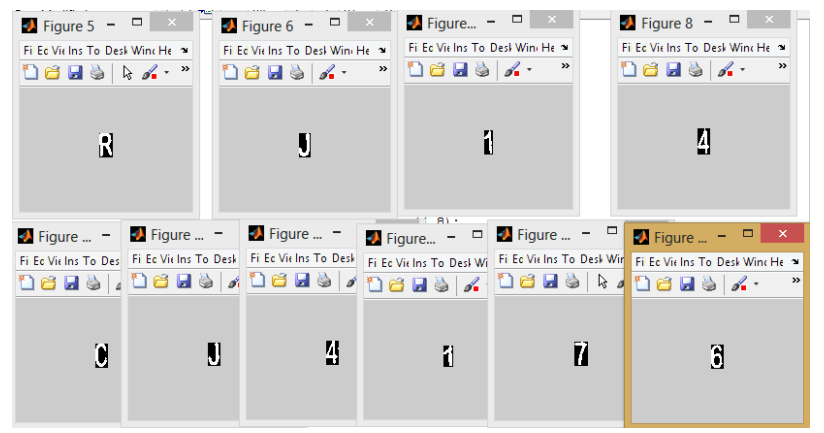

Figure 12. Segmentation of each and every character of the image captured

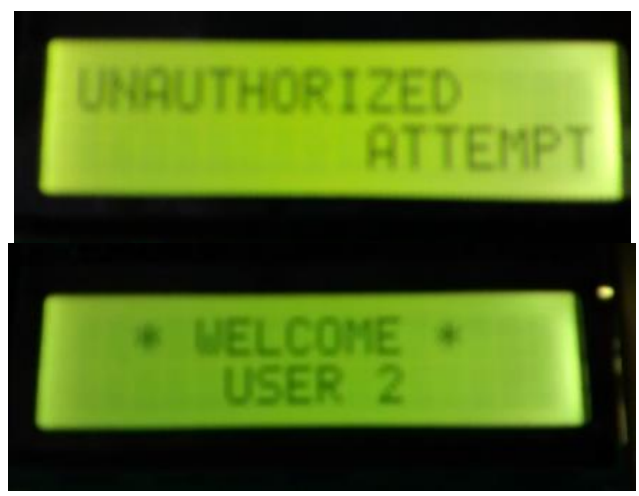

Figure 13. LCD display of unauthorized and authorized user

The data of the vehicle number plate and driver name along with the date and time were stored in an excel sheet for further references.

The whole experiment was conducted by capturing different number plates, which are clear as well as dirty based on the mining conditions. The results showed that the number plate recognition was good when the number plates were clean as shown in Table 2. The camera was place at different distances and the efficiency was shown in Table 3. As the driver fingerprint may not be clean all the time, so the experiment was conducted by gathering clean and dirty fingerprints and the results were shown in Table 4.

Table 2. Efficiency of the number plate recognition

\begin{tabular}{cc}
\hline Parameter & Obtained efficiency \\
\hline Clean number plate & $76.67 \%$ \\
Dusty number plate & $73.33 \%$ \\
\hline
\end{tabular}


Table 3. Efficiency of the number plate recognition at different distances

\begin{tabular}{cc}
\hline Distance & Efficiency for each distance \\
\hline 1 meter & $80 \%$ \\
2 meters & $80 \%$ \\
3 meters & $76.67 \%$ \\
4 meters & $73.33 \%$ \\
5 meters & $73.33 \%$ \\
6 meters & $70 \%$ \\
\hline
\end{tabular}

Table 4. Efficiency of the fingerprint module

\begin{tabular}{cc}
\hline Parameter & Obtained efficiency \\
\hline Clean finger impression & $96.7 \%$ \\
Dusty finger impression & $80 \%$ \\
\hline
\end{tabular}

\section{LIMITATIONS AND SCOPE OF WORK}

Some limitations were observed while implementing the system in mines. As the mine environment is dusty in mines, the number plates will be covered with dust and the number plate recognition rate will be deflected. The drivers hand should be clean for the biometric fingerprint identifications and this will not be accurate if their hands have dust. The number of monitoring points is to be installed, increases with the mining area.

In future, the GPS module can be installed in trucks for tracking the trucks. To ensures, whether the truck has reached the desired destination or not. Face recognition technology also can be implemented to identify the driver.

\section{CONCLUSIONS}

Intelligent unauthorized transportation control uses license plate recognition and biometric fingerprint system. A hardware module was developed for experimental studies using ATMEGA16 microcontroller, DC motor for gate control, webcam for ANPR and biometric fingerprint R305 module. Vehicle license number plate was captured using a digital camera and the characters of the number plate were segmented using Gabor filter. The fingerprint scans the finger and matches with the template created at the time of fingerprint registration at the machine. The output of both ANPR and biometric system were matched with the database and then the microcontroller will activate the opening of the check gate. The checkpoint gate will be closed or opened based on the output provided by the microcontroller and the authenticity of the vehicle and the driver was verified and displayed on LCD. The experimental results showed that ANPR was $80 \%$ and $73.33 \%$ accurate for clean and dustyfaded number plates respectively. The biometric analysis showed $96.7 \%$ and $80 \%$ accuracy for clean and dirty fingerprint respectively.

\section{REFERENCES}

[1] Trading Economics. India GDP from mining. http://www.tradingeconomics.com/india/gdp-frommining, accessed on Jan 17, 2015.

[2] ENVIS. Curbing illegal mining, transport and storage of minerals top
http://www.ismenvis.nic.in/ViewGeneralLatestNews.as px?Id=5256, accessed on Dec. 19, 2015.

[3] MAHADGM. Regulations of minerals. http://www.mahadgm.gov.in/PDF/REGULATION_OF _MINERALS.pdf, accessed on Aug. 22, 2016.

[4] First Post. Coal India plans biggest tech overhaul in 4 decades to check rampant theft. http://www.firstpost.com/business/coal-india-plansbiggest-tech-overhaul-in-4-decades-to-check-rampanttheft-2581686.html, accessed on Jan. 4, 2017.

[5] NCL. http://ncl.gov.in/page.php, accessed on Mar. 17, 2016.

[6] EssenRFID. Major national coal mine adopts RFIDbased vehicle tracking system. http://www.essenrfid.com/Mailer/SECL_Mining.pdf, accessed on Feb. 21, 2016.

[7] Ozbay S, Ercelebi E. (2005). Automatic vehicle identification by plate recognition. World Academy of Science, Engineering and Technology 9(41): 222-225.

[8] Ravi J, Raja KB, Venugopal KR. (2009). Fingerprint recognition using minutia score matching. International Journal of Engineering Science and Technology 1(2): 3542.

[9] Kulkarni P, Khatri A, Banga P, Shah K. (2009). Automatic Number Plate Recognition (ANPR) system for Indian conditions. In: 19th International Conference Radioelektronika'09.

[10] Gnanasivam P, Muttan S. (2010). An efficient algorithm for fingerprint preprocessing and feature extraction. Procedia Computer Science 2: 133-142.

[11] Zhai X, Benssali F, Ramalingam S. (2010). License plate localisation based on morphological operations. In: 11th International Conference on Control Automation Robotics \& Vision.

[12] Bana S, Kaur DD. (2011). Fingerprint recognition using image segmentation. International Journal of Advanced Engineering Sciences and Technologies 5(1): 12-23.

[13] Kolour HS, Shahbahrami A. (2011). An evaluation of license plate recognition algorithms. International Journal of Digital Information and Wireless Communications 1(1): 247-253.

[14] Asthana S, Sharma N, Singh R. (2011). Vehicle number plate recognition using multiple layer back propagation neural networks. International Journal of Computer Technology and Electronics Engineering 1(1): 35-38.

[15] Sharma C, Kaur A. (2011). Indian vehicle license plate extraction and segmentation. International Journal of Computer Science and Communication 2(2): 593-599.

[16] Gill R, Kaur N. (2012). Indian Vehicle number plate detection by image processing technique in Matlab. International Journal of Computer Science and Technology 3(2): 365-367.

[17] Rani PS, Prasad V. (2012). License plate character segmentation based on pixel distribution density. International Journal of Engineering Science and Advanced Technology 2: 1539-1542.

[18] Aggarwal AK, Aggarwal AK. (2013). Vehicle registration plate recognition system based on edge transition by row and column profile on still image. International Journal of Engineering Research and Technology 2(1): 1-8.

[19] Patel C, Shah D, Patel A. (2013). Automatic Number Plate Recognition system (ANPR): A survey. International Journal of Computer Applications 69(9): 
21-33.

[20] Karthikeyan V, Vijayalakshmi VJ, Jeyakumar P. (2013). License plate segmentation using connected component analysis. Journal of Electronics and Communication Engineering 4(5): 18-24.

[21] Sonavane S, Khade A, Gaikwad VB. (2013). Novel approach for localization of indian car number plate recognition system using support vector machine. International Journal of Advanced Research in Computer Science and Software Engineering Research 3(8): 179183.

[22] Mohammad F, Anarase J, Shingote M, Ghanwat P. (2014). Optical character recognition implementation using pattern matching. International Journal of Computer Science and Information Technologies 5(2): 2088-2090.

[23] Kaur A, Ameeta B. (2014). Minutiae extraction and variation of fast fourier transform on fingerprint recognition. International Journal of Engineering Research and General Science 2: 72-84.

[24] Kaur H, Garg NK. (2014). Number plate recognition using Neural Network Classifier and KMEAN. International Journal of Advanced Research in Computer Science and Software Engineering 4(8): 429-434.

[25] Chouhan PS, Govindan VK. (2014). Localization of license plate using characteristics of alphanumeric characters. International Journal of Computer Science and Information Technologies 5(3): 3407-3409.

[26] Sharma R. (2015). Automatic license plate based smart vehicle validation \& security by gate control \& email send. International Journal of Computer Science and Information Technologies 6(2): 952-957.

[27] Kumar VS, Meeravali DS, Deepika G. (2015). A novel model of fingerprint authentication system using Matlab. International Journal of Scientific Engineering and Technology Research 4(17): 3264-3269.

[28] Rajput TS. (2015). Automatic vehicle plate recognition using morphological edge detection and segmentation. International Journal of Emerging Technology and Advanced Engineering 5(1): 500-504.

[29] Otsu N. (1979). A threshold selection method from graylevel histograms. IEEE Transactions on Systems, Man, and Cybernetics 9(1): 62-66.

[30] Hu P, Zhao Y, Yang Z, Wang J. (2002). Recognition of gray character using gabor filters. In: Proceedings of the Fifth International Conference on Information Fusion.

[31] Chaurasia OP. (2012). An approach to fingerprint image pre-processing. International Journal of Image, Graphics and Signal Processing 4(6): 29-35. 